\title{
SOBRE EL USO DE HEURÍSTICAS COMO POSIBLE SOLUCIÓN DEL PROBLEMA DE MARCO
}

\author{
María InÉs SILENZI \\ CONICET \\ Universidad Nacional del Sur \\ misilenzi@gmail.com \\ RODRIGO MORO \\ CONICET \\ Universidad Nacional del Sur \\ rmoro@uns.edu.ar
}

RESUMEN: Se ha propuesto (y criticado) el uso de heurísticas como una herramienta para solucionar el problema de marco. Los objetivos de este trabajo son proveer una clarificación de la literatura filosófica sobre el tema e intentar resolver los debates pendientes considerando la evidencia empírica disponible. Luego de distinguir varios aspectos del problema de marco, analizaremos las disputas filosóficas sobre el tema. A continuación comentaremos la literatura sobre la evidencia empírica relevante proveniente de la psicología cognitiva. Argumentaremos que las heurísticas pueden ser útiles para solucionar sólo un aspecto del problema de marco, aun con varias restricciones.

PALABRAS CLAVE: ciencias cognitivas, clarificación de la literatura, resolución de debates, evidencia empírica, psicología cognitiva

SUMMARY: The use of heuristics has been proposed (and criticized) as a tool to solve the frame problem. The goals of this paper are to provide a clarification of the philosophical literature and to attempt to solve the remaining debates by considering the available empirical evidence. After distinguishing various aspects of the frame problem, we analyze the philosophical disputes around the issue. We, then, discuss the relevant empirical evidence from cognitive psychology. We argue that the heuristics can be useful to solve only one aspect of the frame problem and this solution will be limited by several restrictions.

KEY WORDS: cognitive sciences, literature clarification, debate resolution, empirical evidence, cognitive psychology

\section{Introducción}

Sin lugar a dudas, uno de los problemas con mayor impacto y trascendencia dentro de las ciencias cognitivas es el problema de marco. Es notorio que aun más de 40 años después de su planteamiento inicial, continúa siendo centro de numerosos debates. Tal vez el que no se hayan superado dos de sus dificultades claves (las llamaremos dificultad definicional y dificultad resolutiva) explican su permanencia. En efecto, al examinar la literatura sobre el problema de 
marco, se puede observar, por un lado, que la mayoría de las veces se menciona, especialmente al principio de cada tratamiento particular del problema, la complicación de definirlo. Ésta es la dificultad definicional. Pero no sólo la dificultad definicional del problema de marco ha sido motivo de debate, también lo ha sido la posibilidad de solucionarlo, es decir, ha sido polémica su dificultad resolutiva. El objetivo de este trabajo no es enfocarnos en detalle en cada una de estas dificultades; simplemente queremos hacer notar la relación que se establece entre ambas: antes de evaluar cualquier solución que intente resolver el problema de marco, es necesario aclarar previamente la interpretación particular que de este problema se tenga en mente. Apoyándonos en la literatura, consideraremos el problema de marco como un conjunto de problemas relacionados con la determinación de relevancia. Más importante, distinguiremos entre varios aspectos del problema, lo cual será útil al momento de revisar la literatura sobre el tema.

Estas observaciones, referidas a las dificultades inherentes al problema de marco y a las distintas aristas (aspectos) que el problema de marco conlleva, han sido motivo de extensos y animosos debates filosóficos, que aún hoy perduran. Para dilucidar algunos de estos desacuerdos, nuestro primer objetivo es proveer una clarificación de la literatura filosófica sobre el tema. Sin embargo, aunque creemos que es urgente atender las disputas filosóficas mencionadas, este trabajo se orienta especialmente hacia la disputa filosófica actual sobre la aptitud del uso de heurísticas para proveer una solución al problema de marco. En efecto, si bien se acepta sin problemas que el uso de heurísticas permite dar cuenta de cómo las personas razonan y toman decisiones, no hay acuerdo entre los filósofos acerca de si el uso de heurísticas permitiría resolver el problema de marco (véase, por ejemplo, Samuels 2004, Carruthers 2006a y Fodor 2008). Así, el segundo objetivo de nuestro trabajo es proporcionar una clarificación de dicho debate filosófico. Finalmente, creemos que para brindar una opinión informada sobre este tema, uno debe analizar en detalle la evidencia empírica relevante, proveniente de la psicología cognitiva. Este análisis suele estar ausente en la literatura filosófica y creemos que uno de los principales aportes de este trabajo es proveer dicho análisis. Así, nuestro tercer y último objetivo es intentar resolver la disputa sobre la aptitud de las heurísticas para resolver el problema de marco, considerando la evidencia empírica disponible. De esta manera, la misma disputa se analizará desde un punto de vista filosófico y empírico. Finalmente, y a la luz de lo visto, argumentaremos que 
las heurísticas pueden ser útiles para solucionar sólo un aspecto del problema de marco, aun con varias restricciones.

Vistos nuestros objetivos, el artículo está organizado de la siguiente manera. En la sección 2 se propondrá la interpretación del problema de marco de interés y sus principales aspectos: el aspecto computacional, el epistemológico y el explicativo. En la sección 3, atendiendo ya la disputa de interés en este trabajo, revisaremos la literatura filosófica sobre la aptitud de las heurísticas por resolver el problema de marco y, particularmente, el debate entre Jerry Fodor, Richard Samuels y Peter Carruthers. En la sección 4, y continuando con esta misma disputa, pero ya dentro del área de la psicología cognitiva, se analizará la aptitud resolutiva de las heurísticas, considerando la evidencia empírica disponible. En la sección 5, presentamos posibles objeciones a los resultados de nuestro análisis e intentamos brindar una respuesta para cada una de ellas. Finalmente, presentaremos nuestras conclusiones en la sección 6.

\section{Interpretación del problema de marco y sus principales aspectos}

De acuerdo con la teoría computacional de la mente, a las representaciones mentales se les almacena como símbolos y los procesos mentales son operaciones computacionales sobre esos símbolos. Ahora bien, supóngase que un agente posee una cantidad desmesuradamente amplia de información (algo muy plausible si se piensa en agentes humanos) y debe realizar una tarea que requiere utilizar parte de esa información. Tomaremos la interpretación del problema de marco como refiriendo a un conjunto de problemas que tienen que ver con la manera en que dicho agente selecciona la información relevante de ese vasto conjunto de tal manera que logra realizar la tarea en cuestión.

Una gran cantidad de investigadores del área estarían de acuerdo en concebir, como lo hacemos nosotros, el problema de marco como un problema de relevancia (Glymour 1987; Crockett 1994; Dreyfus 1979; Schneider 2007; Pinker 2005; Shanahan 2009; Ludwing y Schneider 2008, entre otros). Si bien no hay un acuerdo generalizado acerca de cómo concebir el problema de marco, esto no implica que no haya cierta tendencia a entenderlo como un problema de determinación de relevancia. Sirva como ejemplo la siguiente cita de Glymour: "los casos particulares del problema de marco son todos de la siguiente forma: dada una enorme cantidad de cosas y cierta tarea a realizar utilizando algunas de esas cosas, ¿qué cósas son relevantes para la tarea?" (Glymour 1987, p. 65). ${ }^{1}$

\footnotetext{
${ }^{1}$ Las traducciones de todas las citas de este artículo son nuestras.
} 
Incluso Fodor coincidiría plenamente en que la determinación de relevancia es una parte crucial del problema de marco. Aunque él probablemente también quiera agregar otras actividades de los sistemas centrales (como la abducción y la confirmación de hipótesis), sin duda, aceptaría esta actividad como uno de los desafíos centrales del problema de marco. Esta interpretación del problema de marco implica, entonces, un conjunto de problemas (aspectos) interrelacionados con la determinación de relevancia.

Estas observaciones, referidas las distintas aristas (aspectos) que el problema de marco conlleva, han sido motivo de confusión, conduciendo a debates que aún hoy perduran. Con el fin de evitar estas confusiones, lo cual creemos es una tarea necesaria e indispensable a la hora de tratar el problema de marco, a continuación intentaremos dilucidar cada uno de estos aspectos por separado. Tales distinciones serán claves para el análisis posterior que realizaremos sobre la disputa de interés en este trabajo.

\subsection{El aspecto computacional del problema de marco}

El aspecto computacional involucra el supuesto de que la teoría computacional de la mente requiere que los procesos mentales sean computacionalmente tratables, es decir, que sean especificables en términos algorítmicos. Aunque también requiere que estos procesos no demanden ni más tiempo ni más recursos que lo que, se supone, los humanos demandarían. Este aspecto comprende dos dificultades distintas, a las cuales denominaremos dificultad de la vastedad de la información y dificultad de la contextualidad de la relevancia, ambas desarrolladas por Fodor (1986, 1991, 2000, 2008). La dificultad de la vastedad de la información puede verse claramente si se considera la siguiente estrategia ingenua para resolver el problema de marco: analizar cada una de las piezas de información del sistema y evaluar, en cada caso, si es o no relevante para realizar la tarea en cuestión. Claramente, esta estrategia no es factible de aplicar cuando la cantidad de información que posee un agente es abrumadoramente vasta, como es el caso de los seres humanos. Así, para resolver el aspecto computacional del problema de marco uno debe especificar, en primer lugar, qué algoritmos pueden permitir una extracción de la información relevante dentro de una vasta base de datos y con recursos computacionales limitados.

La dificultad de la contextualidad (Fodor la llama de la globalidad) se basa en que la relevancia de una pieza de información depende del contexto; en otras palabras, el hecho de que cierta pieza 
de información sea relevante para realizar una tarea depende del resto de información que se posea. El siguiente ejemplo ilustra el problema. Considérese una tarea mundana como estimar el tiempo que le llevará a uno viajar hasta el trabajo. La información sobre si la selección femenina de hockey de Argentina (conocida como "Las Leonas") gana el mundial de hockey parece, en principio, completamente irrelevante para dicha tarea. Sin embargo, si se cree adicionalmente que, de acuerdo con los noticieros, si Las Leonas ganan el mundial de hockey, se cerrará la avenida central por donde pasa el colectivo para ir al trabajo, entonces el resultado del partido de hockey sí será relevante. Esto parece relativamente simple, pero el problema aparece cuando se intenta trasladar el carácter contextual de la determinación de relevancia a nivel de algoritmos de manipulaciones de símbolos. Más detalladamente, cada representación mental tiene una contrapartida simbólica y los algoritmos de procesamiento de información justamente deben establecerse como manipulaciones de estos símbolos. El problema es cómo lograr una simbolización que sea sensible al contexto informacional. Así, para resolver el aspecto computacional del problema de marco también se deben establecer algoritmos que sean sensibles a los distintos contextos informacionales.

\subsection{El aspecto epistemológico del problema de marco}

Imagínese la siguiente situación. A un agente se le dio una tarea a realizar, para lo cual, dados sus recursos temporales y cognitivos finitos, analizó solamente una parte de la información de su base de datos, la procesó, retuvo parte de ella, desechó otra parte y considera que está listo para realizar la tarea en cuestión. A partir de esta situación, surgen distintas preguntas. En primer lugar, ¿̨cómo sabe el agente que la información retenida es genuinamente relevante si, en principio, el conocimiento de la información no considerada podría convertir la información retenida en irrelevante? A su vez, surge la pregunta de cómo saber que la información desechada es genuinamente irrelevante si, en principio, el conocimiento de la información no analizada podría convertir la información rechazada en relevante. Finalmente, surge la pregunta de cómo sabría el agente que ya ha considerado suficiente información para realizar la tarea si justamente desconoce la información no analizada. En otras palabras, la clave está en cómo sabe el agente en qué punto de la búsqueda de información debe detenerse. Así, cuándo detenerse, cuándo parar de recolectar información, resulta una tarea extremadamente difícil dada la vasta cantidad de información que se puede examinar. El 
algoritmo computacional no puede ir mucho más allá al decidir si lo que computó como relevante ya es suficiente, pues mirar más allá requeriría seguir computando. De alguna manera, sobre la base de información que ya tiene el agente, debería decidir cuándo "hasta acá es suficiente" ("enough is enough", Glymour 1987, p. 70).

Este problema surge porque la relevancia no se puede determinar a priori, y cualquier información puede, en principio, dado el conjunto apropiado de creencias, volverse relevante. Estos planteamientos dan lugar al aspecto epistemológico de la relevancia. El aspecto epistemológico del problema de marco se cuestiona, entonces, cómo un sistema cognitivo "sabe" después de una búsqueda parcial lo que es relevante y que, además, sabe que la información recolectada ya le es suficiente para llevar a cabo una tarea determinada. Es a este aspecto al que se refiere Fodor (1991) cuando caracteriza el problema de marco como el problema de Hamlet: "el problema de marco es el problema de Hamlet, a saber, cuándo parar de pensar” (p. 26). ${ }^{2}$

\subsection{El aspecto explicativo del problema de marco}

Finalmente, no hay que olvidar que este problema se trata en el seno de las ciencias cognitivas. Y el objetivo final de estas ciencias es lograr buenos modelos explicativos y predictivos de cómo funciona de hecho la mente humana. Teniendo este objetivo en cuenta, es importante recalcar que la literatura del área acepta que las personas determinen qué es relevante para una tarea dada de manera rápida y adecuada, aunque, por supuesto, de vez en cuando surjan errores. A veces se falla porque las demandas cognitivas son altas o los recursos cognitivos bajos, pero generalmente los seres humanos determinan la relevancia con la prontitud y precisión, exhibiendo así cierta eficiencia al determinar relevancia. No parece plausible atribuir al azar la prontitud y el nivel de éxito y, por lo tanto, una pretendida solución al problema de marco debería dar cuenta de cómo los humanos determinan eficientemente la relevancia. En otras palabras, se debe explicar cómo a las personas parece "venírsele a la mente" solamente la información relevante para una tarea de entre una cantidad desmesurada de información que poseen, y eso en el momento preciso y de manera adecuada.

\footnotetext{
${ }^{2}$ Desde el comienzo del primer acto de la obra de Shakespeare, Hamlet tiene que vengar la muerte de su padre. Pero durante los siguientes cuatro actos, hasta el desenlace mortal de la última escena, intenta negárselo a sí mismo, lo intelectualiza todo; su cerebro está en constante conflicto con su corazón. No deja de pensar y pensar. Es por estas razones que Fodor utiliza la metáfora de Hamlet para referirse al aspecto epistemológico del problema de marco.
} 
Teniendo en cuenta las distinciones mencionadas, el objetivo de la siguiente sección será revisar la literatura filosófica con respecto a una de las disputas actuales alrededor del problema de marco: la aptitud del uso de heurísticas para resolverlo.

Pero antes de comenzar con la revisión de la literatura, es menester realizar un par de aclaraciones previas. En primer lugar, parecería necesario establecer el significado del concepto de "relevancia", ya que dicho término suele considerarse, con justicia, polisémico. Dado que el problema de marco siempre se plantea en el contexto de la realización de una tarea, consideramos que la definición presentada por Hjørland (2010) es especialmente adecuada: "algo $(x)$ es relevante para una tarea $(T)$, si $x$ aumenta la probabilidad de lograr el objetivo $(O)$ que es implicado por $T$ " (p. 229).

Así, la relevancia de cierta de pieza de información dependerá de si contribuye o no a lograr el objetivo implícito en la tarea en cuestión.

En segundo lugar, es conveniente proveer, al menos, un noción aproximada del concepto de "heurística", agregando algún ejemplo de aplicación (en la sección 4 revisaremos la literatura empírica sobre el tema y presentaremos un análisis más detallado). Así, en principio, una heurística es básicamente una regla práctica que, con poca información y procesamiento, permite obtener una respuesta razonable a ciertas tareas. Por ejemplo, dada la tarea de recoger el equipaje en un aeropuerto que se pisa por primera vez, una heurística (usada por los autores con cierto éxito) consiste simplemente en seguir los pasos de algún compañero de vuelo. Por supuesto, no es una estrategia infalible, pero nótese que utilizando poca información, generalmente se logra el objetivo buscado.

Tal vez sea conveniente, a propósito de nuestra afirmación de que el problema de marco es "un conjunto de problemas (aspectos) interrelacionados con la determinación de relevancia", explicar cómo estos aspectos efectivamente se interrelacionan. En otros escritos (Silenzi 2014), realizamos una sistematización de la posición de Fodor, mostrando que los tres aspectos señalados debían ser resueltos, según Fodor, para proveer una solución satisfactoria al problema de marco. Así, si se plantea que el uso de heurísticas puede responder al desafío que Fodor plantea, debería arrojar luz sobre cada uno de los aspectos señalados. Tal afirmación constituye la base de la que partimos, en la segunda parte del trabajo, para evaluar el uso de heurísticas para resolver nuestro problema de interés.

Antes de comenzar con la interrelación de los aspectos, tal vez sea conveniente aclarar que existen diversas maneras de abordar la 
relación entre los aspectos constituyentes: en este sentido, la propuesta que a continuación sugeriremos no es la única posible ni intenta serlo. Como ya lo hemos mencionado, nuestro análisis parte de la revisión de la literatura sobre psicología cognitiva realizada y la recurrente motivación que algunos investigadores, y particularmente Fodor, parece reflejarse al abordar algunos problemas cognitivos y en especial, al problema de marco. En efecto, observamos que el hecho de que el ser humano resuelve fácilmente problemas cognitivos en la vida cotidiana motiva a algunos investigadores a querer explicar los mecanismos cognitivos involucrados y otras cuestiones relacionadas. De entre ellas, y teniendo especialmente en cuenta a Fodor, se ha prestado especial interés al modo en que los seres humanos determinamos la relevancia de manera eficiente. Más particularmente, y en referencia a los aspectos anteriormente mencionados, es el aspecto (3) del problema de marco el que ha impulsado el debate actual y la vigencia de este problema dentro del campo de investigación de las ciencias cognitivas. De esta manera, el aspecto explicativo del problema de marco se convierte en uno de los principales y actuales objetivos de las ciencias cognitivas.

Ahora bien, si atendemos las soluciones tentativas a este aspecto especial del problema de marco, podemos observar que éste no sólo ha captado la atención de los filósofos de la mente, sino también de los investigadores de la inteligencia artificial (Crockett 1994; Dreyfus 1979). Podríamos decir que el objetivo de estos últimos, y con base en el aspecto (3) recién mencionado, es diseñar sistemas que imiten, exactamente o de manera semejante, la manera en que los seres humanos determinamos la relevancia eficientemente. Por supuesto, esta línea de investigación se vincula con la teoría computacional de la mente prevalente en la misma época.

Pero, ¿̇a qué se está haciendo referencia cuando decimos que lo que se quiere imitar es el modo "eficiente" con el que determinamos la relevancia? Se quieren resolver dos cuestiones. Por un lado, lo que se quiere lograr es un algoritmo tal que, frente a una tarea dada, considere sólo la información relevante en un tiempo relativamente breve y sin mayores costos cognitivos. Esta cuestión, al fin de cuentas, se refiere al aspecto computacional de la relevancia (aspecto 1). Visto de esta manera, podríamos sostener entonces que el aspecto computacional (1) resulta ser una respuesta tentativa desde la teoría computacional de la mente al aspecto explicativo (3) antes explicitado. Nótese que este aspecto es de primer orden, en el sentido de ser un aspecto relativo a la realidad, a saber, la manera en que un sistema determina la relevancia. 
Ahora bien, aun asumiendo que el aspecto computacional pueda ser resuelto, quedaría pendiente otra cuestión que requiere solución: cómo es que un sistema cognitivo, luego de determinar la relevancia, "sabe" que lo que determinó es lo correcto. El punto puede entenderse comparando situaciones. Un sistema puede llegar a determinar correctamente la relevancia de casualidad; sin embargo, aquí no hablaríamos de un éxito cognitivo genuino. En una determinación de la relevancia genuinamente exitosa, el agente no sólo determina correctamente la relevancia, sino que sabe que así lo hizo. Nótese que este es un aspecto de segundo orden, ya que versa sobre el resultado de primer orden obtenido en el aspecto computacional. Retomando los aspectos mencionados, y asumiendo que el aspecto computacional se resolvió, es el aspecto epistemológico (2) del problema de marco el que se convierte en un problema de segundo orden, difícil de resolver.

\section{La disputa filosófica sobre el uso de heurísticas y el problema de marco}

A continuación realizaremos una breve revisión de la literatura filosófica sobre la viabilidad del uso de heurísticas para solucionar el problema de marco. Esta disputa es de interés en este trabajo por dos razones: su vigencia y la posibilidad de ser abordada desde distintos análisis. Al respecto, en esta sección nos limitaremos al análisis y debate filosófico que se plantea entre Peter Carruthers, Richard Samuels y Jerry Fodor, debido al impacto y trascendencia que esta discusión tiene actualmente dentro del campo de las ciencias cognitivas.

$\mathrm{El}$ argumento de la "tratatabilidad computacional", que Carruthers y Samuels defienden, sostiene que el uso de las heurísticas podría proveer tratabilidad computacional, superando así las limitaciones computacionales del problema de marco que Fodor resalta. Para éste, la determinación de la relevancia no es computacionalmente fácil pues requiere evaluar grandes cantidades de información con recursos cognitivos y temporales limitados. Pues bien, para Carruthers (2006a, 2006b) y Samuels $(2004,2005,2010)$ el uso de heurísticas constituye el principal candidato para aliviar la carga computacional. En efecto, estos filósofos sugieren que las heurísticas pueden servir como técnicas que delimitarían subconjuntos computacionalmente tratables de información, sugiriendo que Fodor exagera los requerimientos de la tratabilidad computacional. Argumentan que todo tratamiento computacional requiere que los cálculos sean adecuadamente frugales, y que las heurísticas puedan realizar los procesos de 
cálculo frugales. Mediante el empleo de heurísticas, un sistema puede evitar las evaluaciones exhaustivas al determinar la relevancia creando instancias de búsqueda adecuadas y reglas de detención que limiten efectivamente la cantidad de información relevada en sus cálculos y así considerar un subconjunto adecuado de información para las tareas del sistema. En los términos planteados en la sección anterior, lo que el uso de heurísticas resolvería - de acuerdo con Samuels y Carruthers- es la dificultad de la vastedad de información. Adicionalmente, los autores hacen hincapié en que el uso de las heurísticas parecería poder dar cuenta de lo que realmente sucede en nuestra mente cuando determinamos la relevancia de manera relativamente exitosa, es decir, contribuyen a explicar la eficiencia con la que los seres humanos determinamos la relevancia. Esto, a su vez, parecería estar bien respaldado por investigaciones empíricas de psicología cognitiva. En otras palabras, las heurísticas serían una buena herramienta para contribuir a resolver lo que denominamos el aspecto explicativo del problema de marco. En consecuencia, estos argumentos contribuirían a rebatir la idea de la supuesta imposibilidad de resolver el problema de marco.

¿Cuál es la respuesta de Fodor? Básicamente, la respuesta de Fodor (2008) consiste en señalar que, aun cuando se aceptaran los puntos señalados por sus críticos, el problema de marco no está realmente aún resuelto, ya que quedan muchas cuestiones esenciales pendientes. En primer lugar, no sería claro cómo el funcionamiento de las heurísticas puede generar resultados que sean sensibles al contexto, es decir, al resto de información que posee el sistema. En efecto, como veremos en detalle en la siguiente sección, las heurísticas demandan la búsqueda de cierta información específica, la procesan y generan una respuesta. Así, no es en absoluto claro cómo una heurística va a ser sensible a otra información relevante para la tarea en cuestión. Volviendo al ejemplo presentado anteriormente, no es claro cómo una heurística mundana que genere estimaciones de tiempos de viaje va a ser capaz de tener en cuenta la información sobre el mundial de Las Leonas, así como las otras miles de posibilidades de interrupciones, demoras, etc. En otras palabras, no es claro cómo el uso de heurísticas puede superar la dificultad de la contextualidad de la relevancia.

En segundo lugar, Fodor cuestiona la capacidad de las heurísticas de seleccionar cierta información, considerada como relevante, ignorando directamente el resto de la información examinada. Abiertamente, de manera implícita se estaría asumiendo que la información seleccionada es relevante mientras que el resto de la información se 
consideraría irrelevante. A esta capacidad se le conoce como la estrategia de "dejar dormir a los perros" (sleeping dogs strategy). Pero, nuevamente, ¿cómo sabe el sistema cognitivo que esta información es relevante y que además es suficiente para realizar la tarea de manera satisfactoria? ¿Cómo sabe también que la información restante es irrelevante? Como el enfoque heurístico no da ninguna explicación al respecto, no podemos afirmar, según Fodor, que el enfoque heurístico resuelve efectivamente el problema de marco. Más específicamente, el aspecto epistemológico del problema de marco también quedaría pendiente de resolver. Ni Carruthers ni Samuels dan una respuesta satisfactoria a estos desafíos que Fodor plantea. Veamos con más detenimiento los planteamientos que estos autores proponen a la hora de resolver el problema epistemológico y por qué éstos no le resultan convincentes a Fodor.

Fodor, a pesar de otorgarle cierta credibilidad a las heurísticas, no admite que éstas definitivamente resuelvan el problema de marco. Veamos su argumento, expuesto a través de dos críticas y examinemos por qué Fodor cree que las heurísticas no solucionan "adecuadamente" el problema de marco.

La primera crítica considera que apelar al uso de las heurísticas no resuelve el problema de marco más bien lo desplaza. Si las heurísticas realmente resolvieran el problema de marco, supone Fodor, entonces éstas explicarían el mecanismo por medio del cual una heurística determina qué otra heurística se debería aplicar al momento de ofrecerse ciertos inputs. Decidir qué heurística usar implica elegir, a su vez, una heurística, pero ¿cómo "sabe" el sistema cognitivo que esa heurística va a proveer información relevante y suficiente para la tarea en cuestión?, ¿cómo sabe que la aplicación de ésta, y no de otra heurística es "apropiada"? Como el enfoque heurístico no da ninguna explicación al respecto, no podemos afirmar, dice Fodor, que el enfoque heurístico resuelve efectivamente el problema de marco pues, de acuerdo con lo visto, quedaría pendiente resolver lo que hemos denominado el aspecto epistemológico del problema.

Pues bien, esta acusación no afecta por igual a los autores involucrados en esta disputa. En efecto, a Carruthers la acusación fodoriana contra la aptitud de las heurísticas no lo perturba directamente mientras que a Samuels sí. La respuesta que brinda Carruthers frente a la primera crítica fodoriana es concluyente: la manera en que se resolvería qué heurística utilizar es automática. Al entender el sujeto la tarea que tiene que resolver, el uso de la heurística se dispara automáticamente sin necesidad ni de una decisión consciente ni de 
una heurística de orden superior. Pues bien, supongamos, como afirma Fodor, que las heurísticas se aplicaran de manera automática tal como Carruthers lo explica. Si así fuera, entonces, las inferencias que éstas llevarían a cabo serían locales. Pero lo que estamos discutiendo es cómo es que las heurísticas revisan globalmente nuestro vasto conjunto de creencias a la hora de determinar la relevancia. Los procesos mentales que llevan a cabo esta tarea son de tipo global y no local. Las heurísticas sólo se aplican localmente y, en consecuencia, no son aptas para explicar cómo es que los seres humanos revisamos nuestras creencias holísticamente. Lo que Fodor está criticando es la falta de interés, descuido o negación de algunos científicos cognitivos por resolver la dificultad de la contextualidad de la información del problema de marco (esta crítica se aclarará y ejemplificará en la siguiente sección).

Continuando con nuestro esquema, veamos la actitud de Samuels frente a la primera crítica fodoriana. Si bien este autor defiende el alcance de las heurísticas a la hora de resolver el problema de marco, al garantizar tratabilidad computacional, no "barre bajo la alfombra" las limitaciones que éstas conllevan. Admite, como Fodor, que éstas son incapaces de explicar los mecanismos involucrados que describirían cómo sabe un sistema cognitivo que esta información, y no otra, es relevante a la hora de llevar a cabo una tarea. Coincide, al igual que Fodor, en que, frente a este tipo de cuestiones, la ciencia cognitiva tiene mucho camino por recorrer, aunque, a diferencia de éste, es mucho más optimista en cuanto a sus avances. Este autor admite que el problema de marco implica muchos más aspectos que sólo el computacional y cuestiona los alcances y limitaciones del uso de las heurísticas al resolver el problema de marco.

Veamos una segunda crítica fodoriana. Ésta cuestiona la capacidad de las heurísticas de seleccionar cierta información, considerada relevante, ignorando directamente el resto de la información examinada. Abiertamente, de manera implícita se estaría asumiendo que la información seleccionada es relevante mientras que el resto de la información se consideraría irrelevante. Esta capacidad es conocida como la estrategia de "dejar dormir a los perros" (sleeping dogs strategy). Pero, nuevamente, ¿cómo "sabe" el sistema cognitivo que esta información es relevante y que además es suficiente? ¿Cómo sabe también que la información restante es irrelevante? Como las heurísticas tampoco dan ninguna explicación al respecto, no podemos afirmar, diría también Fodor, que las heurísticas resuelven efectivamente el problema de marco, pues queda manifiesta, a través de 
estas críticas, la incapacidad de las heurísticas de resolver el aspecto epistemológico del problema de marco.

Así, Fodor no acepta que las heurísticas resuelvan absolutamente el problema de marco, pues hay un aspecto que no está resuelto: el epistemológico. Este aspecto, supone Fodor, es clave para comprender la naturaleza del problema; de nada sirve que el aspecto computacional esté resuelto si la problemática "esencial" del problema de marco no está considerada ni mucho menos resuelta. Samuels reconoce y acepta el problema, sin embargo, Peter Carruthers lo desdeña, poniendo incluso en duda que se trate de un aspecto esencial a resolver (analizaremos este punto en nuestra sección de discusión).

Hasta aquí hemos atendido la disputa filosófica en torno a si las heurísticas son aptas o no para resolver el problema de marco. Por supuesto, queda mucho más por analizar pero, dados nuestros propósitos, creemos que lo expuesto es suficiente. A continuación evaluaremos la aptitud de las heurísticas en torno a la evidencia experimental disponible. Como dijimos anteriormente, la aptitud de las heurísticas para resolver el problema de marco puede ser abordado desde distintos análisis. Si se observa la literatura sobre el problema de marco, puede observarse que queda pendiente un análisis de tipo empírico. Es nuestro objetivo, comenzar con esta tarea pendiente y ofrecer un análisis alternativo al meramente filosófico.

\section{Heurísticas, problema de marco y evidencia empírica}

Uno de los primeros hechos que sorprende al analizar la literatura empírica, sobre todo después de leer a Fodor al referirse a "el enfoque heurístico", es que no hay un único enfoque heurístico sino dos. En efecto, en la literatura de psicología cognitiva, hay dos programas de investigación rivales que sostienen que las personas razonan y toman decisiones usando heurísticas, el programa de heurísticas y sesgos y el programa de heurísticas rápidas y frugales. ${ }^{3}$ Así, dividiremos nuestra

${ }^{3}$ Desde luego, la afirmación de que se trata de programas genuinamente rivales puede ponerse en duda. En particular, pueden concebirse como variantes de un solo programa que difieren en las interpretaciones que dan a los efectos contextuales en razonamientos y toma de decisiones (Alfano 2014; Fairweather y Montemayor 2014). Por otra parte, nuestra afirmación de que se trata de programas rivales se basa en las propias declaraciones de los protagonistas, en especial, Gigerenzer plantea su posición en manifiesta oposición al programa de heurísticas y sesgos (véase especialmente Gigerenzer 1996 y 2008). Afortunadamente, nuestra argumentación es completamente independiente de esta discusión. En efecto, nosotros intentaremos mostrar que las mismas conclusiones se siguen de analizar cada uno de los enfoques. Si fueran vistos como un único programa de investigación, nuestra argumentación 
exposición de acuerdo con esta distinción, considerando en detalle, por razones de espacio, sólo una de las heurísticas más trabajadas por cada programa.

\subsection{El programa de heurísticas y sesgos}

Este programa fue iniciado por Tversky y Kahneman en los años setenta (véase un compendio en Gilovich, Griffin y Kahneman 2002). Actualmente, el programa defiende el enfoque de procesamiento dual de información. Nuestras mentes son procesadores de información, pero tienen dos sistemas funcionando simultáneamente, en lugar de uno. El sistema 1 sería automático, requiriendo poco esfuerzo cognitivo, de carácter asociativo, de funcionamiento paralelo, de procesamiento opaco. Por otra parte, habría un sistema 2 con las características opuestas: controlado, requiriendo mucho esfuerzo cognitivo, deductivo, serial, autoconsciente. Las heurísticas serían estrategias del sistema 1 .

La idea es que las personas utilizan heurísticas para elaborar juicios y tomar decisiones que involucran situaciones de incertidumbre. La ventaja es que, comparado con modelos normativos, las heurísticas reducen el tiempo y el esfuerzo para realizar juicios y decisiones. La desventaja es que, en determinadas circunstancias o contextos, estas heurísticas conducen a errores sistemáticos o sesgos. Se propone poner la atención en los errores porque, al igual que en las ilusiones ópticas, en los sesgos se aprecia mejor el funcionamiento de los procesos de elaboración de juicios y decisiones. Para lograr apreciar este tipo de investigaciones, es necesario entrar en los detalles. A continuación comentaremos la heurística más trabajada por el programa de heurísticas y sesgos: la heurística de representatividad (representativeness).

En general, las heurísticas se activan al presentar a los participantes con determinadas tareas. En el caso de la heurística de representatividad se postula que se activa ante la tarea de determinar la probabilidad que determinado objeto pertenezca a cierta categoría, por ejemplo que una persona con ciertas características sea de determinada nacionalidad/profesión/inclinación política. Básicamente, para juzgar la probabilidad de que $A$ sea un $B$, la heurística utiliza el grado en que $A$ sea representativo de $B$ o el grado en que $A$ se asemeje a $B$. Más específicamente, según Kahneman y Frederik

sería más sencilla. Así, nuestra posición puede resumirse de la siguiente manera: aun si uno toma los enfoques de Kahneman y Gigerenzer como programas diferentes (lo cual reconocemos como controversial), se mantienen las mismas conclusiones. 
(2002), la heurística consiste en realizar dos sustituciones: 1) la categoría se reemplaza por un prototipo o ejemplar de dicha categoría; y 2) la evaluación de probabilidad se reemplaza por una evaluación de similitud del objeto con el prototipo.

El programa de heurísticas y sesgos postula que el uso de tal heurística permite dar cuenta de varios sesgos de razonamiento y decisión. Considérese, por ejemplo, el sesgo de pasar por alto la tasa base ${ }^{4}$ (base-rate neglect). Los participantes reciben la siguiente información:

- Un panel de psicólogos ha entrevistado a 30 ingenieros y a 70 abogados. Basados en esa información, se han elaborado descripciones cortas de esas 100 personas. Para las siguientes descripciones indique con qué probabilidad cree que la persona es un ingeniero, usando una escala de 0 a 100.

1) Juan es una de la personas entrevistadas pero su descripción se perdió:

- Probabilidad de que sea un ingeniero:

2) Santiago tiene 45 años, es casado con 3 hijos. Es generalmente muy detallista y ambicioso. No le interesan los temas políticos ni sociales y emplea la gran mayoría de su tiempo libre en sus pasatiempos, los cuales incluyen carpintería y la resolución de problemas matemáticos.

- Probabilidad de que sea un ingeniero:

3) Ricardo tiene 30 años. Está casado pero no tiene hijos. De gran inteligencia, parece que tendrá éxito en su campo. Es muy apreciado por sus colegas.

- Probabilidad de que sea un ingeniero:

En el primer caso, ante la ausencia de información específica, la heurística es imposible de utilizar; así, las personas suelen usar la

\footnotetext{
${ }^{4}$ La tasa base de cierto evento es la probabilidad a priori de que ocurra, es decir, aquella probabilidad del evento que no está condicionada con información específica. Por ejemplo, suponiendo que en cierta población, un 1 por ciento de los ciudadanos son políticos, la tasa base de que un individuo de esa población tomado al azar sea político es, justamente, 1 por ciento. Por supuesto, si uno agrega información sobre el individuo en cuestión (p.ej., es abogado, está afiliado a un partido político, etc.) y uno actualiza la estimación de probabilidad, esa estimación sí estará condicionada con información individual y, por lo tanto, será, a diferencia de la tasa base, una probabilidad a posteriori o condicionada.
} 
información de la tasa base dada y responder treinta por ciento. En el segundo caso, las personas parecen utilizar la heurística de representatividad y dado que Santiago parece muy similar al prototípico ingeniero o al menos, parece más cercano al ingeniero prototípico que al abogado prototípico, suelen estimar altos porcentajes, lo cual no es objetable desde el punto de vista normativo. Sin embargo, en el último caso, donde la información dada no apunta a ninguna de las dos profesiones, los participantes parecen aplicar la misma heurística y tienden a responder cincuenta por ciento (Tversky y Kahneman 1974; Plous 1993). Esta estimación sí es reprochable desde el punto de vista normativo. Si la información particular de la descripción no apunta a ninguna de las dos posibles profesiones, los participantes deberían utilizar la tasa base dada.

Consideremos estas tendencias en relación con la determinación de relevancia (lo cual es clave por la interpretación que tomamos del problema de marco). Dada también la concepción de relevancia que adoptamos, es necesario establecer cuál es el objetivo de la tarea asignada a los participantes. En primer lugar, asumamos que el objetivo es proveer una estimación adecuada. En ese caso, la información sobre la tasa base parecería sumamente relevante. ${ }^{5}$ En segundo lugar, asumamos que el objetivo es, más bien, proveer una respuesta informativa que refleje el grado de creencia del participante sobre la profesión del perfil target. En este caso, es perfectamente razonable pasar por alto la tasa base o, en otras palabras, no contribuye a lograr el objetivo en cuestión y, por lo tanto, no sería relevante.

Analicemos, entonces, qué consecuencias se pueden derivar de este breve repaso con respecto a los debates sobre la posibilidad de que el uso de heurísticas solucione el problema de marco. Comencemos por el aspecto computacional. Si bien no está explicitado de tal forma, es claro que la heurística puede presentarse en la forma de un algoritmo con pasos bien delimitados a seguir. Sin entrar en demasiados detalles, sería algo así como:

Ante la tarea de determinar la probabilidad de que un objeto $A$ pertenezca a la categoría $B$, realizar los siguientes pasos:

\footnotetext{
${ }^{5}$ En este sentido, hasta podría ser criticable dar como respuesta un porcentaje muy alto - cercano al cien por ciento - en el primer perfil, ya que la presencia de falsos positivos (i.e., abogados que encajen en el perfil) haría que los porcentajes no sean tan extremos. Por ejemplo, suponiendo que un cincuenta por ciento de ingenieros encajen en el perfil y sólo el cinco por ciento de los abogados lo haga, la probabilidad sería de alrededor del ochenta por ciento, alta pero alejada del cien por ciento.
} 
1. Buscar en la memoria de largo plazo las características de un caso paradigmático de la categoría $\mathrm{B}$.

2. Considerar en detalle las características del objeto $A$.

3. Comparar el grado de semejanza de los puntos 1 y 2 .

4. Brindar como resultado el resultado obtenido en 3 .

Nótese que, dado un algoritmo de tales características, no surge el problema de la vastedad de la información. Simplemente, la heurística dispara una búsqueda de piezas muy precisas de información. Nótese, sin embargo, que ese gran recorte en la recolección de información también implica insensibilidad con respecto al resto de las creencias. Particularmente, es claro que la tasa base puede ser relevante en ciertos contextos, como en el caso del último perfil presentado y la heurística no lo toma en cuenta en absoluto. Así, del uso de heurísticas no se seguiría el carácter contextual de una adecuada determinación de la relevancia. Por lo tanto, la dificultad de la contextualidad de la relevancia que Fodor plantea quedaría sin resolver. ${ }^{6}$ Así, el aspecto computacional del problema de marco, tomado globalmente, no quedaría resuelto por el enfoque heurístico del programa de heurísticas y sesgos.

Examinemos qué pasaría con el aspecto epistemológico del problema de marco. Parece relativamente claro que este enfoque tampoco puede responder a los desafíos planteados por este aspecto. Nótese que en ningún momento la heurística genera como resultado que sólo la información recolectada es relevante y además es suficiente para resolver la tarea en cuestión de manera satisfactoria; más bien, busca determinadas piezas de información, las procesa y genera una

${ }^{6}$ Sin embargo, aquí es necesario hacer una aclaración importante. Recuérdese que el programa de heurísticas y sesgos postula la existencia de un doble procesador de información. Así el sistema 2 podría, en principio, darse cuenta de que la información de la tasa base es relevante para realizar una estimación adecuada y tenerla en cuenta para su estimación final. No obstante eso, nótese que aquí estaríamos yendo mucho más allá del simple postulado que el uso de heurísticas permite para solucionar el problema de marco. Adicionalmente y aun considerando todo el paquete del programa de heurísticas y sesgos, incluído el funcionamiento del sistema 2, todo eso no implicaría que se puede resolver de manera inmediata la dificultad de la contextualidad de la relevancia. El problema es que el funcionamiento del sistema completo debería representarse en términos algorítmicos y, como si eso fuera poco, el algoritmo propuesto debería poder expresarse en términos de manipulación de signos. No queremos decir que esto sea imposible de realizar. Simplemente hacemos notar que es extremadamente difícil y no está, al menos por el momento, respaldado empíricamente. 
estimación, sin plantearse de manera explícita ni aparentemente implícita, la cuestión de la relevancia. Es más, un análisis proveniente del sistema 2 puede concluir, de hecho, que la información sobre la tasa base (que no se tuvo en cuenta) es genuinamente relevante para la tarea en cuestión. Así, ante preguntas del tipo "¿cómo sabe el agente que la información recolectada por la heurística es relevante y el resto irrelevante?", la respuesta es rotunda: simplemente, no lo sabe. De esta manera, el uso de heurísticas de ninguna manera puede resolver el aspecto epistemológico del problema de marco.

Analicemos finalmente las consecuencias sobre el aspecto explicativo del problema de marco. Parecería a priori que este aspecto sería justamente el que el enfoque heurístico debiera resolver de manera más sencilla (al menos, en comparación con los otros aspectos), ya que la intención de los psicólogos cognitivos es justamente averiguar cuáles son los procesos cognitivos que guían el funcionamiento de nuestra mente. Como vimos en la sección anterior, los filósofos suelen asumir que este aspecto lo resolverían las heurísticas (tal vez Samuels (2010) es el más reticente al respecto). Sin embargo, al mirar en detalle la literatura, es claro que se deben marcar ciertos límites y restricciones. En general, se admite que las personas al realizar una tarea suelen determinar qué información es relevante de manera rápida y adecuada. Ahora bien, es claro que las heurísticas permiten respuestas rápidas, al menos en comparación con modelos normativos, ya que realmente utilizan muy poca información y el procesamiento es muy simple. El problema radica en la adecuación de las respuestas. Como vimos en el caso anterior, eso dependerá, en primer lugar, de lo que entendamos que sea el objetivo de la tarea. Si uno considera que el objetivo es proveer estimaciones adecuadas, las heurísticas pueden no ser el mejor camino a seguir. Por otra parte, si uno considera que el objetivo es brindar una respuesta que refleje ciertas creencias sobre el asunto en cuestión, las heurísticas son un excelente medio para ello. En segundo lugar, dependiendo del contexto informacional (por ejemplo, compárese la situación presentada con un caso similar al presentado, pero con cincuenta por ciento de abogados y cincuenta por ciento de ingenieros), una misma heurística puede tender a brindarnos respuestas correctas o respuestas incorrectas. Así, es crucial tener estas consideraciones en cuenta para proporcionar una buena evaluación de las heurísticas como solución del aspecto explicativo del problema de marco. ${ }^{7}$

${ }^{7}$ El lector podría pensar que defendemos una postura consecuencialista con respecto a la adecuación de las heurísticas, y justamente el programa de heurísticas y 
Sin embargo, antes de llegar a conclusiones definitivas, debemos analizar los resultados del otro programa de investigación rival que se enfoca en heurísticas, a saber, el programa de heurísticas rápidas y frugales.

\subsection{El programa de heurísticas rápidas y frugales}

Gerd Gigerenzer lidera un programa rival de investigación que hace muchas críticas metodológicas al programa de heurísticas y sesgos (véase, por ejemplo, Gigerenzer 1996). Si bien Gigerenzer y sus colaboradores concuerdan con el hecho de que las personas utilizan heurísticas para razonamientos y decisiones, se manifiestan en desacuerdo con que el uso de las mismas genere sesgos. En general, este programa ve los sesgos como artefactos de los procedimientos experimentales del programa rival y, en consecuencia, tiene una postura relativamente optimista con respecto a la racionalidad humana. Como resultado de sus investigaciones, el nuevo programa postula un conjunto distinto de heurísticas. Por razones de espacio, sólo presentaremos la más trabajada por el programa, a saber, la heurística denominada "Toma el/la mejor" (Take the best).

Se postula que esta heurística se activa cuando se tiene que decidir cuál de dos objetos es superior al otro con respecto a cierto criterio $\mathrm{y}$ hay pistas sobre diferentes aspectos que sugieren un resultado $\mathrm{u}$ otro. Lo que la heurística hace es determinar cuál de las pistas tiene mayor validez (proporciona mayor cantidad de respuestas correctas) y utilizar solamente dicha pista para tomar la decisión en cuestión. Si dicha pista no genera una respuesta (i.e., hay empate entre los objetos) se considera con mayor validez la segunda pista y así sucesivamente (Gigerenzer et al. 2002). Por ejemplo, considérese la tarea de determinar cuál de entre dos ciudades tiene una mayor de tasa

sesgos presenta una postura no consencuencialista, sino más bien una de tipo normativa, con lo que se conoce como la "visión estándar de la racionalidad" (Samuels et al. 2004). En primer lugar, nosotros no estamos meramente juzgando la adecuación de las heurísticas a secas, sino la adecuación de las heurísticas para determinar relevancia. Y con este aspecto en mente y dado el enfoque de la literatura con respecto a la realización de tareas, tomamos la concepción de relevancia como si apuntara a aquellas piezas de información que contribuyen a satisfacer el objetivo de la tarea en cuestión. Es allí donde entra el aspecto consecuencialista, y creemos que está motivado porque la literatura del área se enfoca en este aspecto. En segundo lugar, con respecto a nuestra afirmación principal acerca de la inadecuación de las heurísticas para determinar la relevancia en ciertos contextos y para ciertos objetivos, es claro que el programa de heurísticas y sesgos va a compartir plenamente esta afirmación, ya que es justamente uno de los principales aportes que realizan sistemáticamente a la literatura de psicología cognitiva. 
de personas-sin-techo. Para contribuir a la decisión, se les da previamente a los participantes información sobre otros pares de ciudades con detalles acerca de dicha tasa en adición a información sobre desempleo, pobreza, refugios públicos, clima, etc., en cada una de las ciudades. Lo que tienden a hacer las personas, entonces, es utilizar la heurística toma la mejor, es decir, determinan cuál de los aspectos tiene mayor poder predictivo y utilizan sólo ese aspecto para tomar la decisión (Newell et al. 2003, Gigerenzer 2008). Análisis formales muestran que en muchos contextos la heurística tiene una efectividad levemente inferior al modelo bayesiano, ${ }^{8}$ pero utiliza mucho menos información, es decir, es más frugal y la procesa de manera más simple, es decir, es más rápida (Gigerenzer et al. 2002). E incluso hay contextos (p.ej., cuando el material de práctica es limitado) donde la heurística predice mejor que los modelos formales (Gigerenzer y Brighton 2009).

Analicemos, entonces, el funcionamiento de la heurística comentada en relación con la determinación de relevancia. Nótese que el ranking de pistas que ordena hacer la heurística puede tomarse como un ranking de relevancia: en efecto, se busca como criterio de decisión la pista que mayor poder predictivo tenga, lo cual puede ser visto como la pista más relevante al momento de hacer una predicción. De todas maneras, nótese que no toma toda la información relevante, para lo cual tendría que analizar todas o casi todas las pistas.

Analicemos, entonces, qué consecuencias pueden derivar de este breve resumen de la heurística toma el/la mejor sobre el debate de la aptitud del uso de heurísticas para solucionar el problema de marco. Lo haremos, nuevamente, analizando cada uno de los aspectos discriminados.

Comencemos por el aspecto computacional. Este programa de investigación de Gigerenzer y compañía se preocupa justamente por expresar las heurísticas en términos algorítmicos. En el caso de la heurística vista, el algoritmo sería:

1. Busca los valores de los objetos de acuerdo con la pistas, pero en orden de validez de dichas pistas. Comienza comparando los valores de los objetos con respecto a la pista que tenga mayor validez.

2. Si uno de los objetos tiene mayor valor que el otro, entonces detiene la búsqueda y pasa al paso 3. De lo contrario, elimina

\footnotetext{
${ }^{8}$ El modelo bayesiano básicamente utiliza toda la información disponible y la integra aplicando el cálculo de probabilidades.
} 
dicha pista y vuelve al paso 1 . Si no encuentra ninguna pista es encontrada, adivina.

3. Predice que el objeto con mayor valor en la pista analizada es superior en el criterio en cuestión (Gigerenzer 2008, p. 32).

Sin embargo, lo mismo que se dijo con respecto al algoritmo de representatividad vale aquí también: básicamente, el algoritmo exige una búsqueda restringida y guidada de información y eso tiene dos consecuencias que son las caras de una misma moneda: por una parte, se evita la dificultad de la vastedad de la información, ya que el agente no debe analizar toda la información, sino sólo una parte muy pequeña y en un orden predeterminado. Por otra parte, el uso de heurísticas hace que no se tenga en cuenta información adicional que puede alterar el resultado, no pudiéndose obtener el carácter contextual de la determinación de relevancia. Veámoslo con un ejemplo. Supongamos que analizando la información previa sobre otras ciudades, se infiere que la pista con mayor poder predictivo para la tasa de personas-sin-techo es el nivel de desempleo. Ahora bien, supongamos que, dado el par target de ciudades $A$ y $B$, una de ellas (digamos, $A$ ) tiene un alto nivel de desempleo y la otra $(B)$ un bajo nivel de desempleo. La heurística analizada funciona de manera automática y genera como resultado que la ciudad $A$ tendrá una tasa mayor de personas-sin-techo. Ahora bien, supongamos ahora que entre toda la información que se da sobre el par de ciudades target se incluye el dato de que en la ciudad $A$ se le otorga a las personas desempleadas una casa para que puedan vivir. Dada esa información adicional, la pista de desempleo no debería considerarse, sin embargo, el funcionamiento automático de la heurística generaría el mismo resultado que en el caso anterior. Así, el uso de heurísticas no podría solucionar, al menos sin agregados adicionales, la dificultad de la contextualidad de la relevancia.

Con respecto al aspecto epistemológico del problema de marco, es claro que este enfoque rival tampoco puede resolverlo. Simplemente, el uso de esta heurística no permite concluir que la información no analizada es irrelevante y la utilizada es la única relevante y suficiente para resolver correctamente el problema en cuestión.

Por último, con respecto al aspecto explicativo del problema de marco, este programa de investigación sí difiere del anterior. Además de ofrecer un conjunto distinto de heurísticas, la posición global es que en general las personas usan estrategias adecuadas para resolver problemas. Así, el supuesto de que las personas tienden a determinar 
la relevancia con rapidez y adecuadamente, encontraría un fuerte apoyo en este enfoque. Más importante, este programa provee evidencia experimental de la gran efectividad de heurísticas como toma el/la mejor, por lo que el uso de esta heurística parece poder integrarse fácilmente a modelos explicativos de eficacia humana en la determinación de relevancia.

\section{Discusión}

El lector podría presentar una objeción a nuestra afirmación de que las heurísticas no parecen poder resolver la dificultad de la contextualidad de la información. Particularmente, uno podría tener en cuenta que las heurísticas han sido postuladas como productos de la evolución. En efecto, Gigerenzer explícitamente defiende esta postura (2008) y el grupo de investigación de Tversky y Kahneman la reconoce, al menos, como compatible con su propio enfoque (Gilovich et al. 2002 , p. 10). Si las heurísticas fueran producto de la adaptación de nuestros antepasados al ambiente (lo cual es ciertamente plausible), se esperaría que funcionen adecuadamente en la mayoría de los contextos donde se suelen aplicar. En consecuencia, uno esperaría que si hay cierto tipo de información que suelen pasar por alto (por ejemplo, información acerca de la tasa base en tareas de estimación de probabilidades), eso no debería generar grandes problemas de predicción. En otras palabras, las condiciones de laboratorio que generan errores sistemáticos serían muy artificiales y se darían muy rara vez (o tal vez nunca) en condiciones reales (fuera del laboratorio). La razón es que, si la heurística generara frecuentemente errores de predicción, no habría sido seleccionada en primer lugar. Así, el problema de la contextualidad de la información no tendría graves consecuencias en la práctica, por lo que sería posible ignorarlo. Otra línea de ataque podría estar dada por el hecho de que las personas podrían contar con mecanismos para corregir los resultados del uso de heurísticas (como el sistema 2 para el programa de heurísticas y sesgos). Estas formulaciones son muy plausibles y debería investigárseles en detalle. Sin embargo, es claro que ya no estamos tratando con la simple postulación de que el uso de heurísticas puede solucionar el problema de marco. En efecto, nos estaríamos moviendo hacia formulaciones mucho más complejas que incorporan concepciones evolutivas y/o teóricas adicionales, lo cual requeriría, a su vez, mayores elaboraciones teóricas y evidencia empírica adicional. Resumiendo, con la formulación aislada es claro que no se puede solucionar la dificultad de la contextualidad, lo cual no impide que formulaciones que 
agreguen consideraciones evolutivas u otros desarrollos teóricos puedan lograrlo. Por supuesto, ésta es una fuente de ideas para futuras investigaciones.

El lector también podría plantear si las reglas de detención dentro de las heurísticas no permitirían proveer una solución al aspecto epistemológico del problema de marco. En efecto, este aspecto trata sobre cómo el agente sabe cuándo dejar de buscar información para determinar la relevancia y justamente las reglas de detención indican cuándo dejar de buscar información.

Hay varias razones por las cuales las reglas de detención de las heurísticas no pueden ser la respuesta al aspecto epistemológico del problema de marco. En primer lugar, como mencionamos anteriormente, el aspecto epistemológico alude a un aspecto de segundo orden. El aspecto computacional encargado de establecer cómo se determina de hecho la relevancia es de primer orden, es decir, trata acerca de un aspecto de la realidad. Sin embargo, el aspecto epistemológico trabaja justamente sobre ese resultado de primer orden. En otras palabras, una cosa es determinar la relevancia y otra es saber cómo se hizo o saber si el proceso o el resultado son adecuados. Como comentábamos en la sección anterior, en ningún momento las heurísticas generan como resultado que sólo la información recolectada es relevante y además es suficiente para resolver la tarea en cuestión de manera satisfactoria; más bien, buscan determinadas piezas de información, las procesan y generan una estimación, sin plantearse de manera explícita ni aparentemente implícita, la cuestión de la relevancia. Así, las reglas de detención de las heurísticas, si bien indican cuándo dejar de buscar información, no implican que sólo la información recolectada es relevante y que lo que se ha pasado por alto es irrelevante y, por lo tanto, no pueden proveer el material de primer orden necesario sobre el que se responde al aspecto epistemológico.

Otra objeción vinculada al aspecto epistemológico del problema de marco podría vincularse a la literatura sobre epistemología basada en el enfoque de virtud (virtue epistemology). En efecto, este nuevo enfoque epistemológico toma el conocimiento como un logro, el cual es producto de virtudes o habilidades intelectuales y se refleja en la acción (Greco 2010; Morton 2012). En este ámbito, algunos investigadores defienden la postura de que las heurísticas contribuyen a virtudes intelectuales que, de manera confiable, generan conocimiento, el éxito epistemológico por excelencia (Fairweather y Montemayor 2014; Morton 2014). Así, esta postura podría desafiar nuestra conclusión de que el uso de heurísticas no permite solucionar el aspecto 
epistemológico del problema de marco. La literatura en cuestión es extremadamente interesante, sin embargo, la objeción en la que se basa no parece poder sustentarse después de un análisis detallado. En primer lugar, hay investigadores con este enfoque que desafían que el uso de heurísticas confiablemente conduce a conocimiento (Alfano 2014). En segundo lugar, y más importante, lo que denominamos "aspecto epistemológico" es algo mucho más específico que la capacidad de obtener conocimiento, ya que se trata de la capacidad de saber que se ha obtenido suficiente información relevante para resolver la tarea en juego. Así, es perfectamente posible que, aunque el uso de heurísticas no permita lograr dicha capacidad particular, sí contribuya en general a virtudes intelectuales relacionadas con la obtención de conocimiento.

Hay una objeción más general sobre este punto que merece considerarse. En general, hay cierto acuerdo en que las heurísticas serían mecanismos que funcionan de manera automática, sin que medie un procesamiento consciente de información. Así, parecería extraño plantearse si las heurísticas pueden resolver el aspecto epistemológico que eminentemente requiere un procesamiento de tipo consciente. Es cierto que Fodor lo plantea como uno de los requerimientos fundamentales para resolver de manera satisfactoria el problema de marco. Y se entiende el desiderátum de que en una determinación genuinamente exitosa de relevancia, el agente debería saber que el resultado es correcto. Pero tal vez se trate de un ideal epistemológico que no puedan satisfacer agentes con capacidades cognitivas limitadas como somos los seres humanos. Éste es un punto extremadamente interesante, pero un tratamiento detallado excede las posibilidades de este artículo. Queda así, pendiente, para futuras investigaciones.

\section{Conclusión}

A pesar de las diferencias de los dos enfoques en psicología cognitiva, parece haber ciertos puntos en común que vale la pena recalcar. Aparentemente, la evidencia apunta a que las personas parecen utilizar heurísticas para razonar y decidir. Tales heurísticas son tarea-dependientes, es decir, que la tarea dada determina o influye fuertemente en la heurística que las personas utilizan para resolverla. Hay un acuerdo en que las heurísticas utilizan poca información y la procesan de manera relativamente simple, permitiendo así respuestas rápidas. No habría acuerdo en que, en general, las heurísticas tienden a producir respuestas correctas. Sin embargo, aun el enfoque de heurísticas y sesgos reconoce que, en ciertos contextos o si se toman 
en consideración ciertos objetivos, las heurísticas sí tienden a producir respuestas adecuadas. Así, para algunos contextos y objetivos, entonces, las heurísticas podrían ser tomadas como un buen medio para solucionar el aspecto explicativo del problema de marco.

¿Qué sucede con los otros dos aspectos, el epistemológico y el computacional? Como analizamos detalladamente en cada caso, las heurísticas simplemente no pueden solucionar el aspecto epistemológico, ya que de alguna manera, lo eluden: las heurísticas trabajan de manera automática y no cuestionan si ciertas piezas de información son o no relevantes y/o si son suficientes para resolver la tarea: simplemente buscan la información, la procesan y generan la respuesta con base en ese proceso. Así, en ningún momento, se genera un juicio del tipo "este conjunto de información es el único relevante y suficiente para resolver la tarea". Con respecto al aspecto computacional, el análisis de ambos enfoques nos llevó al mismo resultado: si bien el uso de heurísticas solucionaría la dificultad de la vastedad de la información, no podría, al menos sin agregados adicionales, superar la dificultad de la contextualidad.

Resumiendo, lo que puede sostenerse con apoyo de la evidencia empírica es que las heurísticas parecen contribuir a solucionar sólo el aspecto explicativo del problema de marco. Sin embargo, ahí mismo uno tiene que distinguir entre los dos programas de investigación en psicología cognitiva. En el caso del programa de Gigerenzer de heurísticas rápidas y frugales se encontraría un fuerte acuerdo sobre el fenómeno clave a explicar de la eficiencia humana para determinar la relevancia. En el caso del programa de Tversky y Kahenman de heurísticas y sesgos el acuerdo sería más débil y básicamente se restringiría tomando en cuenta contextos informacionales y/o objetivos por parte de los participantes. Establecer estos matices es necesario si uno pretende tener en cuenta la literatura experimental sobre el tema. ${ }^{9}$

${ }^{9}$ Este trabajo fue subvencionado por la Universidad Nacional del Sur (PGI 24/I219). Queremos también expresar nuestra gratitud hacia dos revisores anónimos, cuyos valiosos comentarios y sugerencias contribuyeron al mejoramiento de una versión previa del artículo. 


\section{BIBLIOGRAFÍA}

Alfano, M., 2014, "Expanding the Situationist Challenge to Reliabilism About Inference", en Fairweather 2014, pp. 103-122.

Carruthers, P., 2006a, The Architecture of The Mind: Massive Modularity and the Flexibility of Thought, Oxford University Press, Oxford.

2006b, "Simple Heuristics Meet Massive Modularity", en P. Carruthers, S. Laurence y S. Stich (eds.), The Innate Mind: Culture and Cognition, Oxford University Press, Oxford, pp. 181-198.

Crockett, L., 1994, The Turing Test and the Frame Problem: AI's Mistaken Understanding of Intelligence, Ablex Publishing Corporation, Nueva Jersey.

Dreyfus, H.L., 1979, What Computers Can't Do: The Limits of Artificial Intelligence, Harper Colophon Books, Nueva York.

Fairweather, A., 2014 (ed.), Virtue Epistemology Naturalized: Bridges Between Virtue Epistemology and Philosophy of Science, Springer, Cham.

Fairweather, A. y C. Montemayor, 2014, "Inferential Abilities and Common Epistemic Goods", en Fairweather 2014, pp. 123-139.

Fodor, J., 2008, LOT 2: The Language of Thought Revisited, Clarendon Press, Oxford.

- - 2000, The Mind Doesn't Work That Way: The Scope and Limits of Computational Psychology, The MIT Press, Cambridge.

—_, 1991, "Modules, Frames, Fridgeons, Sleeping Dogs and the Music of Spheres", en L.J. Garfield (ed.), Modularity in Knowledge Representation and Natural-Language Understanding, The MIT Press, Cambridge, pp. 139-149.

—_, 1986, La modularidad de la mente, Morata, Madrid.

Gigerenzer, G., 2008, Rationality for Mortals: How People Cope with Uncertainty, Oxford University Press, Nueva York.

—_ 1996, "On Narrow Norms and Vague Heuristics: A Reply to Kahneman and Tversky", Psychological Review, vol. 103, no. 3, pp. 592-596.

Gigerenzer, G. y H. Brighton, 2009, "Home Heuristicus. Why Biased Minds Make Better Inferences", Topics in Cognitive Science, vol. 1, no. 1, pp. 107-143.

Gigerenzer, G., J. Czerlinski y L. Martingnon, 2002, "How Good are Fast and Frugal Heuristics?", en R. Elio (ed.), Common Sense, Reasoning and Rationality, Oxford University Press, Nueva York, pp. 148-173.

Glymour, C., 1987, "Android Epistemology and the Frame Problem: Comments on Dennett's Cognitive Wheels", en Z.W. Pylyshyn (ed.), The Robot's Dilemma: The Frame Problem in Artificial Intelligence, Ablex Publishing Corporation, Norwood, pp. 65-77.

Gilovich, T., D. Griffin, y D. Kahneman, 2002, Heuristics and Biases: The Psychology of Intuitive Judgment, Cambridge University Press, Cambridge. 
Greco, J., 2010, Achieving Knowledge: A Virtue-Theoretic Account of Epistemic Normativity, Cambridge University Press, Cambridge.

Hjørland, B., 2010, "The Foundation of the Concept of Relevance", Journal of the American Society for Information Science and Technology, vol. 61, no. 2, pp. 217-237.

Kahneman, D. y S. Frederik, 2002, "Representativeness Revisited: Attribute Substitution in Intuitive Judgment", en Gilovich et al. 2002, pp. 49-81.

Ludwig, K. y S. Schneider, 2008, "Fodor's Challenge to the Classical Computational Theory of Mind", Mind and Language, vol. 23, no. 1, pp. 123-143.

Morton, A., 2014, "Acting to Know-A Virtue of Experimentation", en Fairweather 2014, pp. 195-206.

- 2012, Bounded Thinking: Intellectual Virtues for Limited Agents, Oxford University Press, Oxford.

Newell, B.R., N. Weston, y D.R. Shanks, 2003, "Empirical Tests of a Fast and Frugal Heuristic: Not Everyone 'Takes-the-Best'", Organizational Behavior and Human Decision Processes, vol. 91, no. 1, pp. 82-96.

Pinker, S., 2005, "So How Does The Mind Works?", Mind and Language, vol. 20, no. 1, pp. 1-24.

Plous, S., 1993, The Psychology of Judgment and Decision Making, McGraw-Hill, Nueva York.

Samuels, R., 2010, "Classical Computationalism and the Many Problems of Cognitive Relevance", Studies in History and Philosophy of Science, vol. 41, no. 3, pp. 280-293.

— 2005, "The Complexity of Cognition: Tractability Arguments for Massive Modularity", en P. Carruthers, S. Laurence. y S. Stich (eds.), The Innate Mind: Structure and Contents, Oxford University Press, Oxford, pp. 107-121.

—_, 2004, "Rationality and Psychology", en R. Piers y A.R. Mele (eds.), The Oxford Handbook of Rationality, Oxford University Press, Oxford, pp. 279-300.

Samuels, R., S. Stich, y L. Faucher, 2004, "Reason and Rationality", en I. Niiniluoto, M. Sintonen, y J. Wolenski (eds.), Handbook of Epistemology, Kluwer, Dordrecht, pp. 131-179.

Schneider, S., 2007, "Yes, It Does: A Diatribe on Jerry Fodor's The Mind Doesn't Work, That Way", Psyche, vol. 13, no. 1, pp. 1-15.

Shanahan, M., 2009, The Frame Problem, http://plato.stanford.edu/entries/ frame-problem [fecha de última consulta: 07/07/2013].

Silenzi, M.I., 2014, El problema de marco: alcances y limitaciones de los enfoques postcognitivistas, Ediuns, Bahía Blanca.

Tversky, A. y D. Kahneman, 1974, “Judgment under Uncertainty: Heuristics and Biases", Science, vol. 185, no. 4157, pp. 1124-1131.

Recibido el 22 de julio de 2014; revisado el 23 de diciembre de 2014; aceptado el 12 de febrero de 2015. 